\title{
Reflection of COVID Frontline Worker based on family's experience with COVID infection
}

\author{
Madhusudana Pulaganti*1, M.S.Sreedhar ${ }^{2}$ \\ ${ }^{1}$ Research Scientist-I, Multidisciplinary Research Unit, Covid-19 testing Lab, SVRRGGH, Sri Venkateswara Medical College, Tirupati, \\ AndhraPradesh, India-517507 \\ ${ }^{2}$ Professor of Medicine and Principal, Apollo Institute of Medical Sciences and Research, Chittoor, Andhrapradesh.
}

\section{Abstract:}

Covid-19 pandemic created fear for everyone because no vaccine was available; no treatment protocol for COVID-19 patients was fool proof. In this report the experience of the family of Covid froline workers is described. Dr. Madhusudana is a scientist in a Medical College in Andhra Pradesh and his wife Mrs shobharani works as Nursing Staff in the Medical College . The Hospital is declared a COVID Hospital. Thus, both are front line workers during current COVID-19 Pandemic. This created panic in the family in the same way as in almost every individual in any other family.

In this article the treatment and prophylaxis experience of a family of COVID-19 frontline worker is reported. It is hoped that such reports help other Covid frontline workers to take preventive measures to control the COVID-19 and enhances their confidence in coping with the infection and reduces their stress.

Current diagnosis for COVID-19 in India is by RT-PCR test and, Trunat Rapid test kit. Treatment procedures are different based on the severity of the patient. Mrs. Shobharani tested Covid-19 positive on $11^{\text {th }}$ August 2020. Immediately she rushed to designated covid-Out Patient Department of a COVID Hospital. After evaluation, she opted and was permitted for home quarantine with treatment as per protocol current at that time.

\section{Key words: COVID-19, Quarantine, HCQ, Ivermectin}




\section{Corresponding author}

Madhusudana Pulaganti*1

Research Scientist-I,

Multidisciplinary Research Unit, Covid testing Lab,

SVRRGGH, Sri Venkateswara Medical College,

Tirupati,

AndhraPradesh,

India-517507

mail:mdrumadhtpt@gmail.com 


\section{Reflection of COVID Frontline Worker based on family's experience with COVID infection}

\section{Introduction}

Corona virus disease in India reached 4.2 million with recovered cases of 3.25 million and deaths of 71642 as on 8, September, 2020 [1]. The Government Teaching Hospital at Tirupati in Andhra Pradesh was declared a COVID hospital. Dr. Madhusudana and Mrs. Shobharani have been working as frontline workers in the same Institution, Theirs is a family of An six memberstwo old agepersons of 60 and 68 years of age and two younger children with aged of 9 and 7 years. From March 2020 onward, every day they are in the battle field of Carona pandemic either conducting tests or taking care of the COVID affected patients. It is well known that Covid-19 is transmitted by persons with symptomatic as well as asymptomatic infection by droplets when they sneeze, cough or touch surfaces such as tables, doorknobs and handrails. Other people may become infected by touching these objects or surfaces and when they inadvertently touch their eyes, nose or mouth with their infected hands [2]. The situation is particularly tough for children, pregnant women, old people, those who are already laboring under some sort of mental health conditions or chronic illness, in addition to frontline workers [3, 4]. As per the Indian government guidelines, Andhra Pradesh guidelines, suggestions of professional colleagues, $\mathrm{Mr}$ Dr. Madhusudana and Mrs. Shobharani strictly followed the preventive measures daily [5] viz; use of N95 masks, use of gloves, head caps, use of sanitizers, head bath after covid duties, practice of social distancing in house and out of house etc] due to fear of COVID-19 exposure and contamination in family members. In this context, how Dr. Madhusudana and Mrs. Shobharani tested positive for COVID-19 and how the situation was handled in home quarantine treatment and preventive measures adopted to limit COVID-19 among their family members. 


\section{Diagnostic and treatment protocol}

Mrs. Shobharani developed febrile illness with headache on the night of $9^{\text {th }}$ August 2020 when she returned home after completing her shift duty at emergency (causality) ward. As all family members were residing in the same home, as a precautionary measure, sample of throat secretions were collected very next day morning for RT-PCR Covid-19 test and and kept away from duty. Covid-19 test was reported positive on $11^{\text {th }}$ August 2020. Soon after that, Mrs. Shobharani was taken to Covid-OP and medical checkup was done by a Pulmonary physician; an X-ray chest was taken and as there were no characteristic changes, home quarantine was permitted. CT-Scan could not be done at that that time due to non availability. Mrs. Shobharani was kept in home isolation of a in separate room attached with toilet. She was treated with antimalarial Hydroxychloroquine (HCQ) 400mg orally twice a day [6] on day One followed by 200mg BID 5 days, macrolide antibiotic Azithromycin 500mg once a day, multivitamin containing Zinc, (a preparation intended to serve as a dietary supplement with vitamins, dietary minerals, and other nutritional elements once a day, antipyretic acetaminophen (Paracetamol) $500 \mathrm{mg}$ twice a day, Proton Pump Inhibitor pantaprazole 40mg once a day for five days, parasiticidal Ivermectin $12 \mathrm{mg}$ (used to treat many types of parasite infestations) once a day for three days [7,8], Doxycycline $100 \mathrm{mg}$ (broad-spectrum tetracycline-class antibiotic) twice a day, The same treatment was administered to other adult members of the family and treatment with half of the dose was initiated for the children for five days as prophylaxis. One of the old person had comorbidity of diabetes mellitus and Cerebrovascular disease. Dr. Madhusudana daily use to attend his usual duties, in the laboratory, duly following all precautions Preventive measures like sanitizing the house by using $1 \%$ hypochlorite solution was followed. Particular care was taken

of the areas of possible contamination viz, the floorr, toilet, door steps, door handles, locks, and 
taps in the room where Mrs. Shobharani was kept in isolation and outer areas of house were frequently cleaned $1 \%$ hypochlorite spray was used twice a day to clean the floor of the house [9]. All of them used surgical masks, even while staying in the house and during sleeping also. All of them used to gargle with salt water every day morning for 2 to 3 minutes drink lemon juice (water, lemon, pepper, salt and sugar). My mother made house very clean every day, she use to wash bed sheets and cloths every day. House was cleaned daily with Dettol (Chloroxylenol) in hot water; Clothes were washed and then rinsed and dried under sunlight. They ate healthy diet including eggs; chicken, dry fruits, fruits and vegetables along with usual diet. Third day onward Mrs. Shobharani got severe low back pain. After due consultation she used Diclotal-MR. Incidentally, five days after initiation of treatment, Mrs. Shobharani had her menstrual cycle prematurely, 15 days before due date. After six days all family members were tested for COVID-19 by RT-PCR test and all of them tested negativel. Treatment was stopped for all after except for Mrs. Shobharani. However, preventive measures were continued. Mrs. Shobharani was given Calcium supplements along with above treatment. Seventh day onward we started to take care of her much more by giving she was given extra diet including more liquid in diet [10]. Though she regained appetitie, she was denied a drink of jiggery milk due to fear. She was given ORS-L though. She continued her treatment for nine days; doxycycline for 14 days. After fourteen days repeat Covid-19 RT-PCR test was done which tested negative. Her isolation was terminated on $15^{\text {th }}$ day. The isolation room and toilet were closed for two days. The use of surgical mask was continued. She used to sleep in a separate room up to $18^{\text {th }}$ day. ORS-L (oral rehydration salt) and special healthy diet was continued up to 28 days and now also. She returned to her duties of serving COVID-19 patients. Throughout this period they got moral support from all their colleagues in the Institution and from colleagues in the Profession. This 
experience gave lot of confidence to Mr.Madhusudana to support his family members in times of health crisis.

\section{Conclusion:}

COVID -19 frontline workers are at greater risk of contracting the infection. Following systemic preventive measures and treatment protocol and prophylactic treatment to all family members appears to be prudent to ward of COVID threat. . Sanitization of home, use of surgical masks round the clock, social distancing, isolation of patient, protein rich diet, care of patient, timely medication are all important ingredients to successfully cope up with COVID-19 infection. Fostering confidence, timely diagnosis, treatment, care and counseling, for prevention of spread is important in coping with Covid-19 pandemic. Of course this is lesson for next hundred years or so for preservation and wellbeing of human life. It throws a big challenge to strengthen public health system of all countries.

\section{Declaration of competing interest}

Author does not any conflict of interest

\section{Acknowledgements:}

Author was thankful to Dr. Jayabhaskar C, Dr. Madhavi Kondeti, and Dr.Subba rao, for their extended support in diagnosis and treatment of Covid-19 and prophylactic treatment to my family. Author has heartfull aknowledgements to Dr.M.S,Sreedhar.

\section{References}

1.https://www.google.com/search?q=tirupati+covid+cases\&oq=tirupati + covid + cases\&aqs=chro me..69i57.5607j0j7\& \{google:bookmarkBarPinned $\}$ sourceid=chrome\& \{google:instantExtend edEnabledParameter\} \{google:omniboxStartMarginParameter $\}$ ie=UTF-8. 
2. https://www.who.int/news-room/q-a-detail/q-a-how-is-covid-19-transmitted

3. Ministry of Health \& Family Welfare - Government of India. Minding our Minds during the COVID-19 2020.

4. Cdc, Mental health and coping during COVID-19. https:/www.cdc.gov/coronavir us/2019ncov/daily-life-coping/managing-stress-anxiety.html, 2020. (Accessed 17 April 2020).

5.https://www.mohfw.gov.in/pdf/GuidelinesonpreventivemeasurestocontainspreadofCOVID19in workplacesettings.pdf

6. Ministry of Health \& Family Welfare - Government of India, Guidelines on Clinical Management of COVID 19, 2020.https://www.mohfw.gov.in/pdf/RevisedNational ClinicalMa nagementGuidelineforCOVID1931032020.pdf.

7. Hydroxychloroquine and ivermectin: A synergistic combination for COVID-19 chemoprophylaxis and treatment? Angela Patrì, Gabriella Fabbrocini. 2020. JAAD ONLINE| VOLUME 82, 6,

8. White paper on Ivermectin as a potential therapy for COVID-19. Agam Vora,* V.K. Arora, and D. Behera. Indian J Tuberc. 2020, 67(3): 448-451.

9. https://ncdc.gov.in/WriteReadData/1892s/89168637271584172711.pdf

10.https://www.euro.who.int/en/health-topics/health-emergencies/coronavirus-covid19/publications-and-technical-guidance/food-and-nutrition-tips-during-self-quarantine 